\title{
AN INTUITIONISTIC FUZZY MULTI-EXPERT AND MULTI-CRITERIA SYSTEM FOR EFFECTIVE PERFORMANCE MANAGEMENT
}

\author{
Ahmet BESKESE ${ }^{1^{*}}$, Cengiz KAHRAMAN ${ }^{2}$, Shirli ENDER BUYUKBAY ${ }^{3}$, \\ Faik Tunc BOZBURA ${ }^{4}$ \\ ${ }^{1,4}$ Department of Management Engineering, Faculty of Engineering, \\ Bahcesehir University, Besiktas, 34353, Istanbul, Turkey \\ ${ }^{2}$ Department of Industrial Engineering, Management Faculty, Istanbul Technical University, \\ Besiktas, 34367, Istanbul, Turkey \\ ${ }^{3}$ Institute of Social Sciences, Bahcesehir University, Besiktas, 34353, Istanbul, Turkey
}

Received 8 November 2016; accepted 26 July 2017

\begin{abstract}
Organizations, in their pursuit of accomplishing their vision and goals, need effective management of human resources. Performance Management, among other Human Resources Management (HRM) practices, is the central function, as it delivers the necessary data that complements and enables the other functions. Building an effective performance management system is a multicriteria problem that requires contribution from experts having diverse backgrounds. Moreover, performance management is an inherently vague concept since almost the whole process requires linguistic assessments rather than numerical ones. Hence, to handle all those issues, an intuitionistic fuzzy multi-criteria and multi-expert analytic hierarchy process (AHP) based management model is proposed in this paper. In the determination of the criteria weights of the model, both the aggregated and compromised assessments of the experts are used in order to observe the effects of these two methods on the results. A numerical application is given to illustrate the use of the model.
\end{abstract}

Keywords: aggregated group decisions, compromised decisions, analytic hierarchy process (AHP), human resources management (HRM), intuitionistic fuzzy sets, performance management.

JEL Classification: C65, D70, D81.

\section{Introduction}

Performance management is "a continuous process of identifying, measuring and developing performance of individuals and teams, and aligning performance with the strategic goals of the organization" (Aguinis, 2009). It is important to define an HR system that relates to particular performance criteria (Jiang et al., 2012), because the field of HR system and performance

${ }^{\star}$ Corresponding author. E-mail: ahmet.beskese@eng.bau.edu.tr

This is an Open Access article distributed under the terms of the Creative Commons Attribution License (http://creativecommons. org/licenses/by/4.0/), which permits unrestricted use, distribution, and reproduction in any medium, provided the original author and source are credited. 
management generally has inconsistent conceptualizations, and mostly lack of cumulative body of knowledge (Chadwick, 2010; Lepak, Liao, Chung, \& Harden, 2006). Conceptually, performance management systems have influence on organizational and financial outcomes, as well as contextual factors; such as industry (Datta, Guthrie, \& Wright, 2005) and strategy (Youndt, Snell, Dean, \& Lepak, 1996). Moona, Scullenb, and Lathamc (2016), for example, state the facilitation effect of forced distribution rating systems on task performance through better motivation and attraction of high talent. Buller and McEnvoy's (2012) statement "HRM activities are strategically important because they are the potentially valuable, rare, difficult to imitate and substitute for, and they are central to creating the organizational capability to enact the firm's strategic goals" may be meaningful in defining the importance of performance management system within HRM. Based on 'line of sight' model (Boswell, 2006; Boswell, Bingham, \& Colvin, 2006), Buller and McEnvoy's (2012) further argument is that, "superior performance is hypothesized to result from directly linking strategic goals and action plans to organizational capabilities and culture, job-specific group competencies and norms, and individual knowledge-skills-abilities, motivation and opportunity". It is the employees' understanding of the firm's strategic goals as well as the actions necessary to accomplish the goals that enable superior performance. Moreover, if HRM is applied effectively in an organization, it simultaneously improves the job satisfaction of employees, company image both within and outside of the company, and even market share and the overall performance level of the organization (Altarawneh, 2016).

Performance management system has a broad perspective, covering organizational dimensions, as well as the individual ones; assisting human resource functions for organizational and individual goal achievement (Aguinis, Joo, \& Gottfredson, 2011).

There exist numerous models of performance management process (Armstrong, 2000; Cardy \& Leonard, 2011) which most consist of the activities of performance agreement/ goal setting, performance monitoring/facilitation, performance appraisal and feedback, and improved performance (Gruman \& Saks, 2011). Both (Gruman \& Saks, 2011) and (Aguinis et al., 2011) note that, performance management system should have a holistic perspective, encompassing the entire organization and should cover all components of performance. Nonetheless, Guest (2011) cautions researchers from overly sophisticated research designs and statistical analyses, and addresses some basic questions for the advancement of the field: "How do we measure HRM practices; which practices or combination of practices have the highest impact on performance; and under what conditions do HRM practices make a positive difference?". In light of the gathered literature in the field, this article has a fairly different perspective to an effective performance management system, which might deliver advancement in the field, while addressing some of Guest's (2011) queries.

In this study, authors are proposing a ready-to-use generic performance management model - that meets the needs of all types of organizations; regardless of their size, industry, strategy or culture- that encompasses pivotal performance management characteristics, among which organizations should consider during their performance management processes. However, the methodology provided for use allows customized modifications based on strategical and cultural aspects of the organization.

The second originality of this paper comes from its first time usage of interval-valued intuitionistic fuzzy sets to determine the weights of the main and sub-criteria for an effective 
performance management system in a firm. Hence, particularly at times of uncertainty, the methodology will guide managers in assigning the appropriate weight for each criterion to ensure an effective performance management system.

Literature indicates that there are different levels of analyses and different measures of performance (Paauwe \& Boselie, 2005), however, according to Buller and McEvoy (2012), performance may be measured at three levels, -organizational, group and individual levels, each contributing to the overall performance. The third distinctive originality of this model is that; it provides decision-makers a performance management mindset that is available for use at all three levels of appraisal and analysis. Then, for each level of performance appraisal measure, a single best-prioritized method can be driven, where flexible, adaptable, and nonstandard - but tailor-made method- may be used based on the needs of the individuals, work-teams, business unit, or the whole organization.

The fourth major originality proposed in this model is the mathematical methodology behind decision making among vague criteria. The performance management criteria used are completely intangible and should be assessed by linguistic terms rather than exact numerical assessments. The fuzzy set theory can capture the vagueness included in these linguistic terms and provides excellent tools to handle it. The ordinary fuzzy sets have been recently extended to type-2 fuzzy sets, hesitant fuzzy sets, intuitionistic fuzzy sets, and neutrosophic sets aiming at defining membership functions with more details and reflecting the hesitancy of decisionmakers (Balin \& Baraçli, 2015; Zavadskas, Bausys, \& Lazauskas, 2015; Chen, 2017; Zavadskas et al., 2017). In this paper, interval-valued intuitionistic fuzzy sets are preferred for converting the linguistic assessments to numerical values since they efficiently handle the hesitancy of the decision-makers by comprising both membership and non-membership degrees together and they are the most widely used fuzzy set extension in the recent literature (Wei, Zhao, \& Wang, 2012; Hajiagha, Hashemi, \& Zavadskas, 2013; Yu, 2013; Zhou, Tao, Chen, \& Liu, 2014; Liu, Li \& Antuchevičienè, 2016; Yang, Chen, \& Zhang, 2017).

In the literature, there are two different methods to deal with multi-expert AHP problems. The first is mathematical aggregation of individual ratings, whereas the second is agreement of experts on a compromised rating through discussion sessions. If the former is the case, separate matrices are aggregated by an aggregation operator for interval-valued intuitionistic fuzzy sets. On the other hand, the latter is generally preferred when there is an easy communication and discussion medium among the decision-makers. In most studies, regardless of any reason stated, any one of these two methods is used and the decisions made. However, in cases where they yield different results, counter to random selection, it is advisable to analyze which method is most adequate to the situation at hand and only then to select the method. Therefore, as an additional contribution of this study, results from both methods have been obtained and analyzed to determine whether there is a significant variation.

The rest of the paper is organized as follows. The following first section introduces the pillar characteristics of effective performance management system, and extensively explains their sub-attributes upon which each attribute is based. In the further section, the intuitionistic fuzzy multi-expert and multi-criteria system is introduced. In the application section, the multi-expert aggregated and compromised rating results are obtained and results were drawn based on the proposed performance management model. The paper is concluded with management implications and further research suggestions. 


\section{Attributes for an effective performance management system}

Employees' performance is measured through a systematic process of performance appraisal that is embedded in the performance management system. An effective performance management system facilitates valuing the top performers, encourages communication between employees and managers, establishes a uniform standard for evaluation, and enables the organization to determine its strengths and weaknesses.

As aforementioned, the appraisal is only a part of the performance management system. As a first step, managers need to define the aspects of performance, through determining the individual goals in relation to job analysis. Only later, based on clear indicators, managers may implement some standardized evaluation methods that best suit to the specific unit needs. Finally managers deliver feedback to the appraisees. Feedback is the pivotal aspect that differentiates performance management from performance appraisal, as it leads the process to a next level for advancement and recognition. First of all, the evaluation results facilitate identification of the problems to performance, predicting the areas necessary for training, development, motivation, or modifications; serving to the advancement purpose. Second, the feedback delivers concrete indicators for rewarding, which may result as increase in pay or incentives, promotion, and career opportunities like succession planning; where it serves the administrative purpose.

Organizations of various size, industry, age, or structure have all strategy to pursue, cultural dimension where employees create certain value, and these strategic and contextual factors are tied to the objectivity and functionality of the system. These criteria have been drawn through a brainstorming process within a focus group study, together with a total of five professional and academic experts in the field. All the attributes and sub attributes explained below were drawn at that session. These criteria were confirmed through some one-to-one talk and consultations, where only later the theoretical and academic basis was researched. These confirmed criteria are strategic congruence, contextual congruence, objective implementation, and functionality; which each has some sub attributes that is assumed to constitute an effective performance management system as illustrated in Figure 1 and explained below.

\section{Strategic Congruence (SC)}

An effective performance management model requires to be strategically congruent. Performance management is the backbone in assuring organization's strategies and goals. In that sense, when modeling a performance system congruent with organization's strategies, (1) alignment to the organizational goals, (2) organizational support, and (3) the role of the executive, are the three main important aspects to establish.

Alignment to organizational goals (AOG): When the system is strategically congruent, not only raters and ratees are more confident in the process of performance management, but all parts own the whole process. In essence, 'line of sight' theory (Boswell, 2006; Boswell et al., 2006) - employees' understanding of the firm's strategic goals and the necessary actions to achieve the goals-, and Buller and McEnvoy's (2012) attempt to identify the linkages between firm's strategy, its human resources, and performance outcome, complements the required 


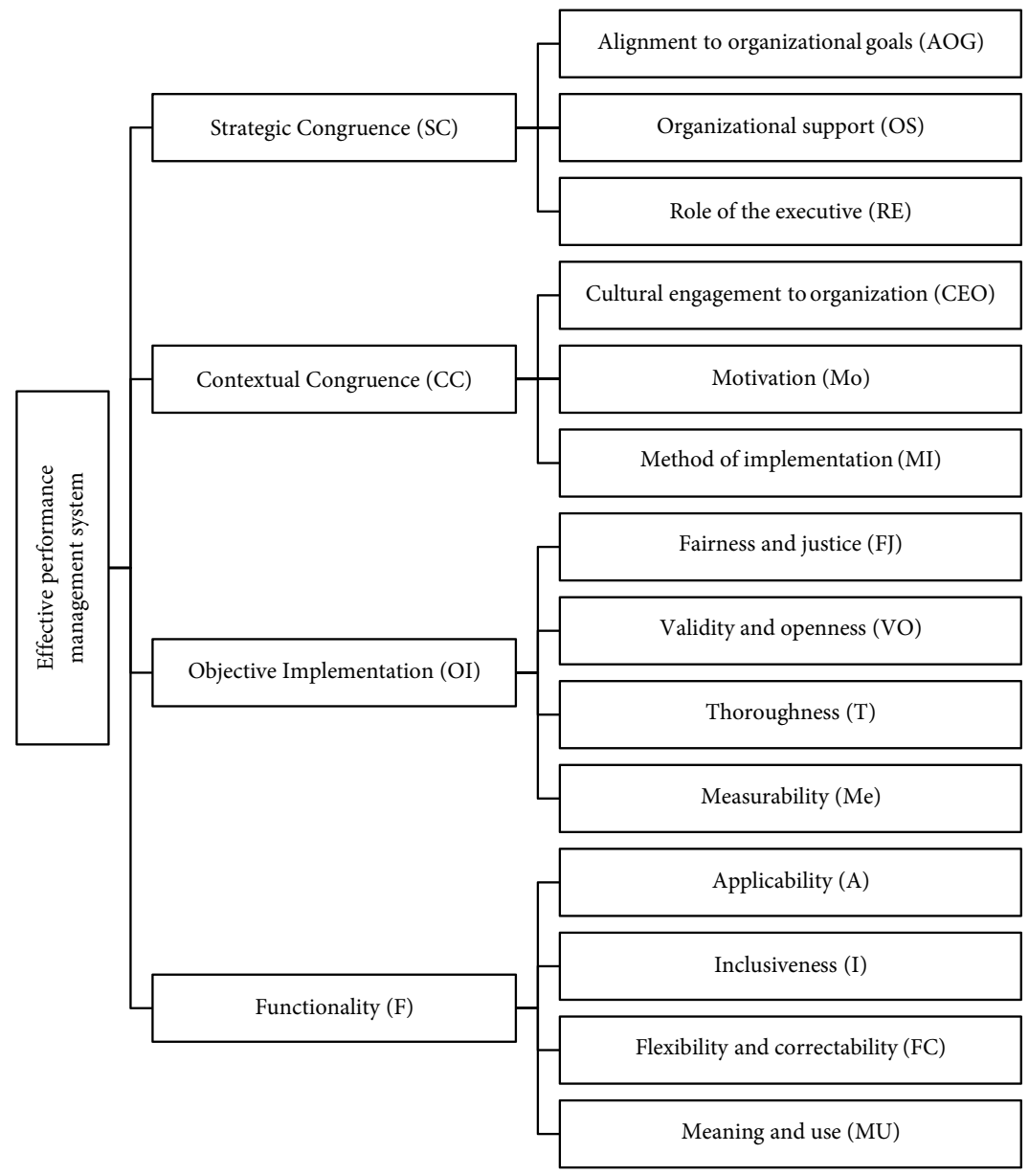

Figure 1. Criteria for an effective performance management system

strategic congruence in the performance management system. Thus, when considering an effective performance management system, it is highly essential to involve strategic aspects of the organization into the whole process.

Organizational support (OS): Additionally, the strength-based performance appraisal and goal setting process, proposed by Bouskila-Yam and Kluger (2011) are two additional essential aspects, which enable the performance management system to be strategically congruent. According to their argument (Bouskila-Yam \& Kluger, 2011), "managers are instructed to design goals for their subordinates that would simultaneously address their unit business goals (interest of the company) and enable expression of subordinates' strengths that would promote subordinates' well-being." This win-win principle, creates both a climate of organizational support over the employee, and also attains a supportive and positive role on the executive. 
Role of the executive ( $R E)$ : Furthermore, the executive is the first hand supporting agent within a supportive organization; hence the role and attitude of the executive is highly crucial in maintaining performance congruent with organization's strategy. Managers should have the ability and skills not to use the performance assessment through 'orthodox' technique, but see it as a remedy to weakness and means for performance development (Bach, 2005). Therefore, the role of the executive, the motivation and value he/she gives on the performance management process determines the effectiveness of the whole performance management system.

\section{Contextual Congruence (CC)}

According to Taylor (2014), “organization's culture is an important conceptual tool that can provide useful insights into the paradox of performance management." Similar to strategic aspect, contextual dimensions impact the efficiency of a performance management system (Aguinis et al., 2011). To enable the performance system congruent with cultural and behavioral aspects of the organization, firms need to assure employees' (1) cultural engagement to the organization and the process of performance management; (2) foster their motivation for their work and the performance system, and finally (3) have a positive perception over the fair and objective method of implementation.

Cultural engagement to organization (CEO): The performance management system should be aligned with organization's culture, reflecting it and enabling an organizational cultural engagement.

Motivation (Mo): Organizations need to be cautious about its aim when constructing a performance management system, as it is a double-edged sword. "The single largest pitfall for performance management system is a negative side-effect which undermines the motivation, morale and behavior of human resources" (Arnaboldi, Lapsley, \& Steccolini, 2015). Mone and London (2010) suggest that designing a performance management process to foster employee engagement will lead to higher levels of performance. To maintain and enhance an effective performance management process, organizations need to focus on employee engagement (Gruman \& Saks, 2011) and promote managers' motivation to the process (Taylor, 2014).

Method of implementation (MI): Nonetheless, the methods chosen for performance assessment are very distinctive in determining contextual congruence. All of the methods Bouskila-Yam and Kluger mention (2011) may affect in establishing contextually congruent system.

\section{Objective Implementation (OI)}

Another highly essential dimension in effective performance management system is objectivity and fairness of the implementation process. Most appraisal processes fail to succeed because of biased ratings, unintentional subjective conclusions, or simply lack of any standard scientific system. Nonetheless, (1) an objective system requires to treat the whole process in fairness and justice; (2) the measurement tools need to be valid and open; (3) the implementation process needs to be such thorough that it is entirely owned by all members of the organization; and finally (4) the results need to be measurable, meaningful and usable. 
Fairness and justice (FJ): Objective implementation is one of the key characteristics of an efficient performance management system. A fair and just assessment that is bias free may be quite optimal; however, for efficient results, resolving objectivity issue is crucially important. According to P. Prowse and J. Prowse (2009), removal of 'top-down' ratings and replacement with multiple-rater reporting -360-degree appraisal- will reduce the subjectivity and inequity of appraisal ratings, gender bias ratings, etc. An objective system is meant to be fair and just (Erdogan, 2002).

Validity and openness (VO): A performance management system needs to be valid and open (Aguinis et al., 2011), where there is an ongoing two-way communication process among supervisor and employee. Therefore, not only the goals and objectives of the system need to be clear and specific, but also the feedback, information exchange and performance standards need to be clearly communicated for an open and valid system.

Thoroughness $(T)$ : Involving all individuals in the evaluation process creates thoroughness in the system (Aguinis et al., 2011). Chytas, Glykas, and Valiris (2011) claim that it is possible for managers to gain insight about how their actions might affect outcomes if they work with models that integrate management debate, communication, dialogue and experimentation as part of performance management system, which may be a powerful communication and learning tool. For this fact, thoroughness may be considered to enable a credible model of performance management system.

Measurability $(\mathrm{Me})$ : A system that aims at serving the strategic, developmental and administrative goals should by all means be constructed on measurable aspects. Performance systems tend to involve biased errors because of the spontaneity and subjectivity aspects. Furthermore, they are observed to lack analytic capability and are insufficient to carry out predictive, measurable modeling. Moreover, measurement tools based on quantifiable indicators deliver results with meaningful, interpretable and usable information.

\section{Functionality (F)}

A strategically and contextually congruent performance management system, as highly objective as can be, needs to be functional. Functionality is last of the major pillars of an effective performance management system. Even if members own the process, have positive attitude, are as objective as can be, for full functioning, the system needs to (1) be applicable and user-friendly, (2) be inclusive, (3) allow flexibility and corrections, and (4) have a sense of meaning and use.

Applicability (A): To define an effective performance management model, it needs to be an applicable and functional system which is accepted and used by all in the organization; if otherwise, the aforementioned negative attitude towards performance appraisal and management will be inevitable.

Inclusiveness (I): The inclusive aspect of the system (Aguinis et al., 2011) is another sub-attribute, which promotes all individuals to get involved and be part of the system. This allows all participants to have a voice in the process of designing and implementing the process.

Flexibility and correctability (FC): A system that is considered to serve strategic needs ought to be flexible, and be open to modifications and corrections (Aguinis et al., 2011). Such a principle enables optimum evaluation opportunity for different situations; meanwhile 
it ensures constant improvement of the process. Therefore, a system open to corrections, especially at challenging times of unjust decisions, may be considered to be highly functional.

Meaning and use (MU): As an important component of the whole system, being meaningful (Aguinis et al., 2011) is another essential characteristic, which brings out interpretable and useful results. Furthermore, in attempt to interpret the results of performance measurement, the cause and effect relationships among measures are mostly a fuzzy mess of interactions and interdependencies. They are always linear and one-way (Chytas et al., 2011). However, the more non-linear or multi-way interactions are, the more meaningful and useful they may become. Furthermore, from the developmental point of view, a useful system, that generates meaningful results will ultimately be establishing learning and growth-based environment.

\section{Methodology using interval-valued intuitionistic fuzzy sets}

\subsection{Interval-valued intuitionistic fuzzy sets}

Intuitionistic fuzzy sets developed by Atanassov (1983) involve both the membership and non-membership grades together for describing any $x$ in $X$ such that the sum of the grades is less than or equal to 1 . In the following, some basic definitions of intuitionistic fuzzy sets are given.

Definition 1. Let $X \neq \varnothing$ be a given set. An intuitionistic fuzzy set in $X$ is an object $\tilde{A}$ given by

$$
\tilde{A}=\left\{\left\langle x, \mu_{\tilde{A}}(x), v_{\tilde{A}}(x)\right\rangle ; x \in X\right\},
$$

where $\mu_{\tilde{A}}: X \rightarrow[0,1]$ for membership and $v_{\tilde{A}}: X \rightarrow[0,1]$ for non-membership satisfy the condition $0 \leq \mu_{\tilde{A}}(x)+v_{\tilde{A}}(x) \leq 1$, for every $x \in X$.

Definition 2. The $\alpha$-cut of an intuitionistic fuzzy set is defined as (Atanassov, 1986)

$$
\tilde{A}_{\alpha}=\left\{x \in X \mid \mu_{\tilde{A}}(x) \geq \alpha, v_{\tilde{A}}(x) \leq 1-\alpha\right\} .
$$

In the literature, there are several aggregation operators developed for the various types of intuitionistic fuzzy sets. In this study, interval-valued intuitionistic fuzzy averaging (IIFA) aggregation operator is used since the experts are assumed to have equal influence on the final decision.

Definition 3. Let $\tilde{\alpha}_{j}=\left(\left[a_{j}, b_{j}\right],\left[c_{j}, d_{j}\right]\right)(j=1,2, \ldots, n)$ be interval-valued intuitionistic fuzzy numbers and let IIFWA: $Q^{n} \rightarrow Q$, if

$$
\operatorname{IIFWA}\left(\tilde{\alpha}_{1}, \tilde{\alpha}_{2}, \ldots, \tilde{\alpha}_{n}\right)=w_{1} \tilde{\alpha}_{1} \oplus w_{2} \tilde{\alpha}_{2} \oplus \ldots \oplus w_{n} \tilde{\alpha}_{n},
$$

then IIFWA is called an interval-valued intuitionistic fuzzy weighted averaging (IIFWA) operator, where $Q$ is the set of all interval-valued intuitionistic fuzzy numbers (IVIFNs), $w=\left(w_{1}, w_{2}, \ldots, w_{n}\right)$ is the weight vector of the IVIFNs $\tilde{\alpha}_{j}(j=1,2, \ldots, n)$, and $w_{j}>0, \sum_{j=1}^{n} w_{j}=1$ (Zhao, Xu, Ni, \& Liu, 2010; Chen, 2014; Zhou et al., 2014). The IIFWA operator can be further transformed into the following form: 
$\operatorname{IIFWA} A_{w}\left(\tilde{\alpha}_{1}, \tilde{\alpha}_{2}, \ldots, \tilde{\alpha}_{n}\right)=\left(\left[1-\left(\prod_{i=1}^{n}\left(1-a_{i}\right)\right)^{w_{i}}, 1-\left(\prod_{i=1}^{n}\left(1-b_{i}\right)\right)^{w_{i}}\right],\left[\left(\prod_{i=1}^{n} c_{i}\right)^{w_{i}},\left(\prod_{i=1}^{n} d_{i}\right)^{w_{i}}\right]\right)$.

If $w=(1 / n, 1 / n, \ldots, 1 / n)$, then the IIFWA operator reduces to an interval-valued intuitionistic fuzzy averaging (IIFA) operator, where

$$
\begin{aligned}
& \operatorname{IIFA}\left(\tilde{\alpha}_{1}, \tilde{\alpha}_{2}, \ldots, \tilde{\alpha}_{n}\right)=\frac{1}{n}\left(\tilde{\alpha}_{1} \oplus \tilde{\alpha}_{2} \oplus \ldots \oplus \tilde{\alpha}_{n}\right)= \\
& \left(\left[1-\left(\prod_{i=1}^{n}\left(1-a_{i}\right)\right)^{1 / n}, 1-\left(\prod_{i=1}^{n}\left(1-b_{i}\right)\right)^{1 / n}\right],\left[\left(\prod_{i=1}^{n} c_{i}\right)^{1 / n},\left(\prod_{i=1}^{n} d_{i}\right)^{1 / n}\right]\right) .
\end{aligned}
$$

\subsection{The steps of the methodology}

The proposed method includes two different approaches (ie. compromised preferences, and aggregated preferences) for multi-expert decision making. The steps of those approaches are given below:

\subsubsection{Initial steps for aggregated preference}

Step 1. Collect the linguistic pairwise comparison matrix of criteria for each expert and convert the linguistic data to their corresponding interval-valued intuitionistic fuzzy sets using Table 1 to obtain individual interval-valued intuitionistic judgment matrix $\tilde{R}$ for each expert.

Table 1. Interval-valued intuitionistic scale

\begin{tabular}{|l|c|}
\hline \multicolumn{1}{|c|}{ Linguistic Terms } & Membership and Non-membership values \\
\hline Absolutely Low $(\mathrm{AL})$ & $([0,0.2],[0.65,0.8])$ \\
\hline Very Low $(\mathrm{VL})$ & $([0.1,0.25],[0.6,0.75])$ \\
\hline Low $(\mathrm{L})$ & $([0.2,0.3],[0.55,0.7])$ \\
\hline Medium Low (ML) & $([0.25,0.35],[0.5,0.65])$ \\
\hline Approximately Equal (AE) & $([0.3,0.6],[0.25,0.4])$ \\
\hline Medium High (MH) & $([0.5,0.65],[0.25,0.35])$ \\
\hline High (H) & $([0.55,0.7],[0.2,0.3])$ \\
\hline Very High (VH) & $([0.6,0.75],[0.1,0.25])$ \\
\hline Absolutely High $(\mathrm{AH})$ & $([0.65,0.8],[0,0.2])$ \\
\hline
\end{tabular}

Let $\tilde{R}=\left(\tilde{r}_{i j}\right)_{n \times n}=\left(\left[\mu_{i j}^{-}, \mu_{i j}^{+}\right],\left[v_{i j}^{-}, v_{i j}^{+}\right]\right)_{n \times n}$ be an interval-valued intuitionistic judgment matrix as follows:

$$
\tilde{R}=\left[\begin{array}{ccc}
\left(\left[\mu_{11}^{-}, \mu_{11}^{+}\right],\left[v_{11}^{-}, v_{11}^{+}\right]\right) & \cdots & \left(\left[\mu_{1 n}^{-}, \mu_{1 n}^{+}\right],\left[v_{1 n}^{-}, v_{1 n}^{+}\right]\right) \\
\vdots & \ddots & \vdots \\
\left(\left[\mu_{n 1}^{-}, \mu_{n 1}^{+}\right],\left[v_{n 1}^{-}, v_{n 1}^{+}\right]\right) & \cdots & \left(\left[\mu_{n n}^{-}, \mu_{n n}^{+}\right],\left[v_{n n}^{-}, v_{n n}^{+}\right]\right)
\end{array}\right]
$$


The reciprocal value of $\left(\left[\mu_{i j}^{-}, \mu_{i j}^{+}\right],\left[v_{i j}^{-}, v_{i j}^{+}\right]\right)$in $\tilde{R}$ is $\left(\left[v_{j i}^{-}, v_{j i}^{+}\right],\left[\mu_{j i}^{-}, \mu_{j i}^{+}\right]\right)$. For instance, the reciprocal value of $\left(\left[\mu_{n 1}^{-}, \mu_{n 1}^{+}\right],\left[v_{n 1}^{-}, v_{n 1}^{+}\right]\right)$is $\left(\left[v_{1 n}^{-}, v_{1 n}^{+}\right],\left[\mu_{1 n}^{-}, \mu_{1 n}^{+}\right]\right)$.

For exactly equal, $\mathrm{EE}=([0.5,0.5],[0.5,0.5])$ is assigned with an exactly same reciprocal value.

Step 2. Aggregate the interval-valued intuitionistic fuzzy pairwise comparison matrices using Eq. (5) to obtain the aggregated interval-valued intuitionistic judgment matrix $\tilde{R}_{g}$.

$$
\tilde{R}_{g}=\left[\begin{array}{ccc}
\left(\left[\mu_{g_{11}}^{-} \mu_{g_{11}}^{+}\right],\left[v_{g_{11}}^{-}, v_{g_{11}}^{-}\right]\right) & \cdots & \left(\left[\mu_{g_{1 n}}^{-} \mu_{g_{1 n}}^{+}\right],\left[v_{g_{1 n}}^{-}, v_{n}^{-}\right]\right) \\
\vdots & \ddots & \vdots \\
\left(\left[\mu_{g_{n 1}}^{-}, \mu_{g_{n 1}}^{+}\right],\left[v_{g_{n 1}}^{-}, v_{g_{n 1}}^{-}\right]\right) & \ldots & \left(\left[\mu_{g_{n n}}^{-} \mu_{g_{n n}}^{+}\right],\left[v_{g_{n n}}^{-}, v_{g_{n n}}^{-}\right]\right)
\end{array}\right] .
$$

\subsubsection{Initial steps for compromised preference}

Step 1. Moderate a discussion of experts to end up with the compromised linguistic pairwise comparison matrix of criteria.

Step 2. Convert the linguistic data to their corresponding interval-valued intuitionistic fuzzy sets using Table 1 to obtain compromised interval-valued intuitionistic judgment matrix $\tilde{R}_{c}$.

Let $\tilde{R}=\left(\tilde{r}_{i j}\right)_{n \times n}=\left(\left[\mu_{i j}^{-}, \mu_{i j}^{+}\right],\left[v_{i j}^{-}, v_{i j}^{+}\right]\right)_{n \times n}$ be an interval-valued intuitionistic judgment matrix as follows:

$$
\tilde{R}_{c}=\left[\begin{array}{ccc}
\left(\left[\mu_{g 11}^{-}, \mu_{11}^{+}\right],\left[v_{g 11}^{-}, v_{g 11}^{+}\right]\right) & \cdots & \left(\left[\mu_{g 1 n}^{-}, \mu_{g 1 n}^{+}\right],\left[v_{g 1 n}^{-}, v_{1 n}^{+}\right]\right) \\
\vdots & \ddots & \vdots \\
\left(\left[\mu_{g n 1}^{-}, \mu_{g n 1}^{+}\right],\left[v_{g n 1}^{-}, v_{g n 1}^{+}\right]\right) & \cdots & \left(\left[\mu_{g n n}^{-}, \mu_{g n n}^{+}\right],\left[v_{g n n}^{-}, v_{g n n}^{+}\right]\right)
\end{array}\right] .
$$

The reciprocal value of $\left(\left[\mu_{i j}^{-}, \mu_{i j}^{+}\right],\left[v_{i j}^{-}, v_{i j}^{+}\right]\right)$in $\tilde{R}_{c}$ is $\left(\left[v_{j i}^{-}, v_{j i}^{+}\right],\left[\mu_{j i}^{-}, \mu_{j i}^{+}\right]\right)$. For instance, the reciprocal value of $\left(\left[\mu_{n 1}^{-}, \mu_{n 1}^{+}\right],\left[v_{n 1}^{-}, v_{n 1}^{+}\right]\right)$is $\left(\left[v_{1 n}^{-}, v_{1 n}^{+}\right],\left[\mu_{1 n}^{-}, \mu_{1 n}^{+}\right]\right)$.

For exactly equal, $\mathrm{EE}=([0.5,0.5],[0.5,0.5])$ is assigned with an exactly same reciprocal value.

\subsubsection{Common steps}

Step 3: Calculate the score judgment matrix $\tilde{S}=\left(\tilde{s}_{i j}\right)_{n \times n}$ and the interval multiplicative matrix $\tilde{A}=\left(\tilde{a}_{i j}\right)_{n \times n}$.

The score judgment matrix of $\tilde{R}_{g}$ and $\tilde{R}_{c}$ is represented by the matrix $\tilde{S}=\left(\tilde{s}_{i j}\right)_{n \times n}=\left[\mu_{g_{i j}}^{-}-v_{g_{i j}}^{+}, \mu_{g_{i j}}^{+}-v_{g_{i j}}^{-}\right]$as follows:

$$
\tilde{S}=\left[\begin{array}{ccc}
{\left[\mu_{g_{11}}^{-}-v_{g_{11}}^{+}, \mu_{g_{11}}^{+}-v_{g_{11}}^{-}\right]} & \cdots & {\left[\mu_{g_{1 n}}^{-}-v_{g_{1 n}}^{+}, \mu_{g_{1 n}}^{+}-v_{g_{1 n}}^{-}\right]} \\
\vdots & \ddots & \vdots \\
{\left[\mu_{g_{n 1}}^{-}-v_{g_{n 1}}^{+}, \mu_{g_{n 1}}^{+}-v_{g_{n 1}}^{-}\right]} & \cdots & {\left[\mu_{g_{n n}}^{-}-v_{g_{n n}}^{+}, \mu_{g_{n n}}^{+}-v_{g_{n n}}^{-}\right]}
\end{array}\right] .
$$



lows:

The interval multiplicative matrix $\tilde{A}=\left(\tilde{a}_{i j}\right)_{n \times n}=\left[10^{\left(\mu_{g_{i j}}^{-}-v_{g_{i j}}^{+}\right)}, 10^{\left(\mu_{g_{i j}}^{+}-v_{g_{i j}}^{-}\right)}\right]$is given as fol-
ows:

$$
\tilde{A}=\left[\begin{array}{ccc}
{\left[10^{\left(\mu_{g_{11}}^{-}-v_{g_{11}}^{+}\right)}, 10^{\left(\left(\mu_{g_{1 n}}^{+}-v_{1 n}^{-}\right)\right)}\right]} & \ldots & {\left[10^{\left(\mu_{g_{i j}}^{-}-v_{g_{i j}}^{+}\right)}, 10^{\left(\mu_{g_{i j}}^{+}-v_{g_{i j}}^{-}\right)}\right]} \\
\vdots & \ddots & \vdots \\
{\left[10^{\left(\mu_{g_{n 1}}^{-}-v_{g_{n 1}}^{+}\right)}, 10^{\left(\left(\mu_{g_{n 1}}^{+}-v_{n 1}^{-}\right)\right)}\right]} & \ldots & {\left[10^{\left(\mu_{g_{n n}}^{-}-v_{g_{n n}}^{+}\right)}, 10^{\left(\mu_{g_{n n}}^{+}-v_{g_{n n}}^{-}\right)}\right]}
\end{array}\right]
$$

Step 4: Determine the priority vector of the interval multiplicative matrix by calculating the $\tilde{w}_{i}$ interval for each criterion using Eq. (11).

$$
\tilde{w}_{i}=\left[\frac{\sum_{j=1}^{n} \tilde{a}_{i j}^{-}}{\sum_{i=1}^{n} \sum_{j=1}^{n} \tilde{a}_{i j}^{+}}, \frac{\sum_{j=1}^{n} \tilde{a}_{i j}^{+}}{\sum_{i=1}^{n} \sum_{j=1}^{n} \tilde{a}_{i j}^{-}}\right] .
$$

Step 5: Construct the possibility degree matrix $P=\left(p_{i j}\right)_{m \times n}$ by comparing the obtained weights in the previous step. Possibility degree reflects the probability of one fuzzy number is larger than another fuzzy number and can be used to compare two fuzzy numbers. To do this, use Eq. (12) (Xu \& Da, 2003).

$$
P\left(w_{i} \geq w_{j}\right)=\frac{\min \left\{L_{w_{i}}+L_{w_{j}}, \max \left(w_{i}^{+}-w_{j}^{-}, 0\right)\right\}}{L_{w_{i}}+L_{w_{j}}}
$$

where $L_{w_{i}}=w_{i}^{+}-w_{i}^{-}$and $L_{w_{j}}=w_{j}^{+}-w_{j}^{-}$and $p_{i j} \geq 0, p_{i j}+p_{j i}=1, p_{i i}=1 / 2$.

Step 6: Prioritize the $P=\left(p_{i j}\right)_{m \times n}$ by Eq. (13).

$$
w_{i}=\frac{1}{n}\left[\sum_{j=1}^{n} p_{i j}+\frac{n}{2}-1\right] \text { for } i=1,2, \ldots, m \text {. }
$$

Step 7: Normalize the weights vector obtained in Step 6 to give $w_{i}^{T}$ for $i=1,2, \ldots, m$.

The flowchart of the aforementioned methodology is illustrated in Figure 2.

\section{An illustrative example:}

A human resources manager wants to determine the weights of four performance criteria. These criteria are initiative, adaptability, leadership, and teamwork. Let the initial linguistic pairwise comparison matrix be as in Table 2 .

In Table 3, the corresponding intuitionistic fuzzy numbers are substituted into the pairwise comparison matrix in Table 2. 


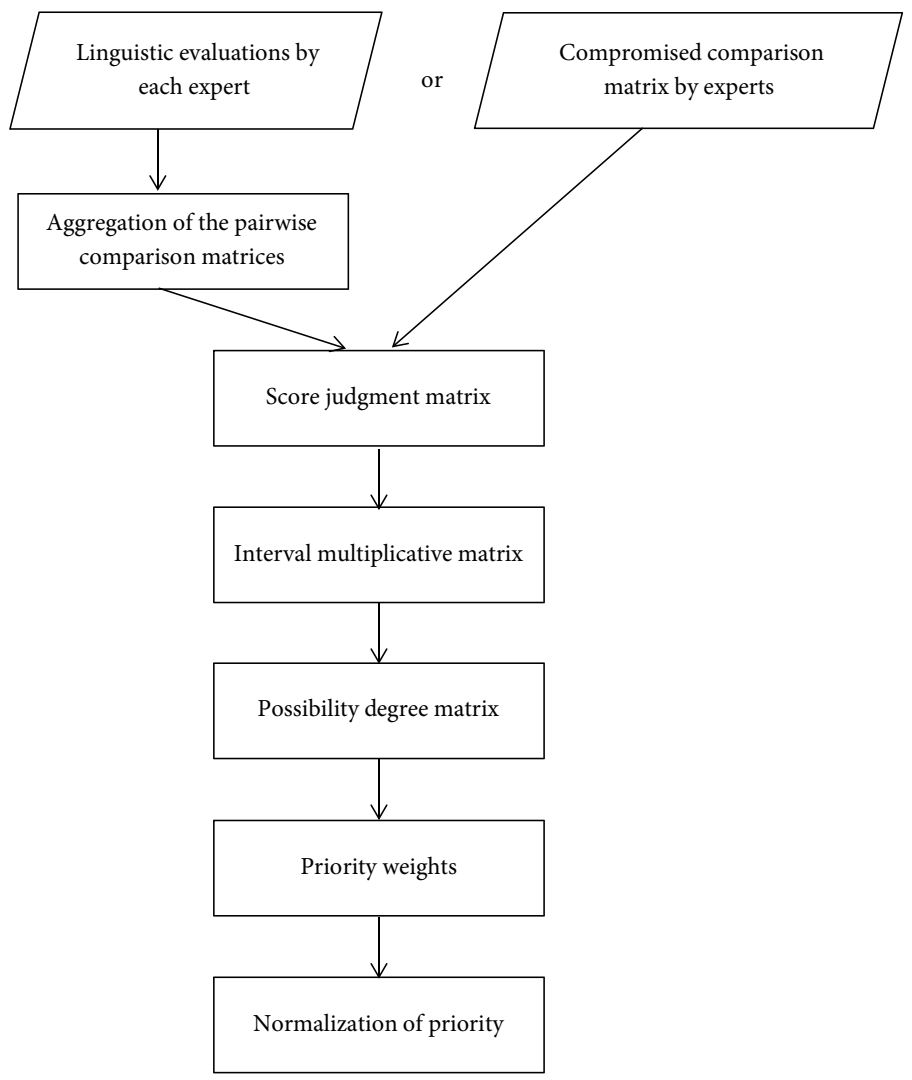

Figure 2. Flow chart of the proposed method

Table 2. Initial linguistic pairwise comparison matrix

\begin{tabular}{|l|c|c|c|c|}
\hline & Initiative & Adaptability & Leadership & Teamwork \\
\hline Initiative & $\mathrm{EE}$ & $\mathrm{AE}$ & $\mathrm{VL}$ & $\mathrm{AL}$ \\
\hline Adaptability & & $\mathrm{EE}$ & $\mathrm{L}$ & $\mathrm{VL}$ \\
\hline Leadership & & & $\mathrm{EE}$ & $\mathrm{ML}$ \\
\hline Teamwork & & & & $\mathrm{EE}$ \\
\hline
\end{tabular}

Table 3. Pairwise comparison matrix with corresponding intuitionistic fuzzy numbers

\begin{tabular}{|l|c|c|c|c|}
\hline & Initiative & Adaptability & Leadership & Teamwork \\
\hline Initiative & $([0.5,0.5],[0.5,0.5])$ & $([0.3,0.6],[0.25,0.4])$ & $([0.1,0.25],[0.6,0.75])$ & $([0,0.2],[0.65,0.8])$ \\
\hline Adaptability & & $([0.5,0.5],[0.5,0.5])$ & $([0.2,0.3],[0.55,0.7])$ & $([0.1,0.25],[0.6,0.75])$ \\
\hline Leadership & & & $([0.5,0.5],[0.5,0.5])$ & $([0.25,0.35],[0.5,0.65])$ \\
\hline Teamwork & & & & $([0.5,0.5],[0.5,0.5])$ \\
\hline
\end{tabular}


The score judgment matrix is obtained as in Table 4 .

Table 4. The score judgment matrix

\begin{tabular}{|l|c|c|c|c|c|c|c|c|}
\hline & \multicolumn{2}{|c|}{ Initiative } & \multicolumn{2}{c|}{ Adaptability } & \multicolumn{2}{c|}{ Leadership } & \multicolumn{2}{c|}{ Teamwork } \\
\hline Initiative & 0 & 0 & -0.1 & 0.35 & -0.65 & -0.35 & -0.8 & -0.45 \\
\hline Adaptability & -0.35 & 0.1 & 0 & 0 & -0.5 & -0.25 & -0.65 & -0.35 \\
\hline Leadership & 0.35 & 0.65 & 0.25 & 0.5 & 0 & 0 & -0.4 & -0.15 \\
\hline Teamwork & 0.45 & 0.8 & 0.35 & 0.65 & 0.15 & 0.4 & 0 & 0 \\
\hline
\end{tabular}

The interval multiplicative matrix is obtained as in Table 5.

Table 5. The interval multiplicative matrix

\begin{tabular}{|l|c|c|c|c|c|c|c|c|}
\hline & \multicolumn{2}{|c|}{ Initiative } & \multicolumn{2}{c|}{ Adaptability } & \multicolumn{2}{c|}{ Leadership } & \multicolumn{2}{c|}{ Teamwork } \\
\hline Initiative & 1.000 & 1.000 & 0.794 & 2.239 & 0.224 & 0.447 & 0.158 & 0.355 \\
\hline Adaptability & 0.447 & 1.259 & 1.000 & 1.000 & 0.316 & 0.562 & 0.224 & 0.447 \\
\hline Leadership & 2.239 & 4.467 & 1.778 & 3.162 & 1.000 & 1.000 & 0.398 & 0.708 \\
\hline Teamwork & 2.818 & 6.310 & 2.239 & 4.467 & 1.413 & 2.512 & 1.000 & 1.000 \\
\hline
\end{tabular}

Applying the remaining four steps, the weights of the performance criteria are found to be as follows:

Initiative: 0.183; Adaptability: 0.156; Leadership: 0.306; Teamwork: 0.355.

\section{Weighing criteria for an effective performance management system}

To help managers in building an effective performance management system, the confirmed main and sub-criteria defined in Section 2 need to be prioritized. Such a decision necessarily requires a multi-expert multi-criteria analysis. In multi-expert decision making, experts' opinions can either be collected individually and then aggregated, or obtained as a compromised assessment after a series of discussion sessions. Those two different methods may yield different results that can directly affect the final decision of the managers. Considering this, the analysis in this study has been carried out in two phases: (1) individual rating and later analysing the aggregated data; and (2) coming to a consensus through a face-to-face discussion and analysing the mutually driven (compromised) data. The rating team is comprised of three experts in the field of human resources management: the first is a human resources (HR) director in a corporate organization; the second is an academician with a corporateexperience in HR management; and the third is an academician working in the field of HR management and specifically on performance management. The selection of these experts was made on the basis of collaborating both the practice and academic perception, so as to obtain optimum possible result. The availability of both professional background and a researcher's mind-set facilitates unbiased judgment. Furthermore, years of experience together with degree of involvement in performance appraisal and management process have been determining criteria on the selection of the involved experts. 
Upon communicating the project with the selected experts, at first hand the individual rating and aggregation was carried. The matrices for individual ratings have been digitally sent to all three experts. Each independently filled in the matrices. Later, the study leader digitally collected all ratings and aggregated them through combining them into decision matrices. The results of the first phase have been analysed and interpreted below in Section 3.1.

The three experts were then invited to a series of meetings where they mutually decided through a discussion session of evaluating each criterion again by pair-wise comparisons. To eliminate any loss of creativity, prior to mutual agreement sessions, experts were encouraged with enough time to have their individual analysis on the criteria. Meanwhile, the expert selection was on the basis of a mutually spoken language, so as to overcome any communication barriers, and to ensure that all discussions are clear for all. Furthermore, a moderator facilitated the sessions, so as to balance any dominant member influencing the group decision. Later, the study leaders analysed the data and interpreted as explained below in Section 3.2.

\subsection{Analysis based on aggregated assessments}

Step 1. The linguistic pairwise comparison matrices of main and sub-criteria for each expert are collected as in Table 6. Those matrices are converted to their corresponding intervalvalued intuitionistic fuzzy sets using Table 1.

Step 2. The interval-valued intuitionistic fuzzy pairwise comparison matrices are aggregated using Eq. (5) to obtain the aggregated interval-valued intuitionistic judgment matrices in Table 7. Here, an interval-valued intuitionistic fuzzy weighted averaging (IIFWA) operator is used.

Table 6. Linguistic assessments of three experts

\begin{tabular}{|c|c|c|c|c|}
\hline \multicolumn{5}{|c|}{ Expert 1 } \\
\hline wrt goal & SC & CC & OI & F \\
\hline SC & EE & MH & L & H \\
\hline CC & & EE & L & MH \\
\hline OI & & & EE & VH \\
\hline F & & & & EE \\
\hline
\end{tabular}

\begin{tabular}{|c|c|c|c|c|}
\hline \multicolumn{5}{|c|}{ Expert 2 } \\
\hline wrt goal & SC & CC & OI & F \\
\hline SC & EE & H & MH & MH \\
\hline CC & & EE & ML & ML \\
\hline OI & & & EE & MH \\
\hline F & & & & EE \\
\hline
\end{tabular}

\begin{tabular}{|c|c|c|c|c|}
\hline \multicolumn{5}{|c|}{ Expert 3 } \\
\hline wrt goal & SC & CC & OI & F \\
\hline SC & EE & VH & MH & AE \\
\hline CC & & EE & L & VL \\
\hline OI & & & EE & ML \\
\hline F & & & & EE \\
\hline
\end{tabular}

\begin{tabular}{|c|c|c|c|}
\hline wrt SC & AOG & OS & RE \\
\hline AOG & EE & VH & AE \\
\hline OS & & EE & L \\
\hline RE & & & EE \\
\hline
\end{tabular}

\begin{tabular}{|c|c|c|c|}
\hline wrt SC & AOG & OS & RE \\
\hline AOG & EE & VH & MH \\
\hline OS & & EE & ML \\
\hline RE & & & EE \\
\hline
\end{tabular}

\begin{tabular}{|c|c|c|c|}
\hline wrt SC & AOG & OS & RE \\
\hline AOG & EE & VH & H \\
\hline OS & & EE & L \\
\hline RE & & & EE \\
\hline
\end{tabular}

\begin{tabular}{|c|c|c|c|}
\hline wrt CC & CEO & Mo & MI \\
\hline CEO & EE & H & VL \\
\hline Mo & & EE & AL \\
\hline MI & & & EE \\
\hline
\end{tabular}

\begin{tabular}{|c|c|c|c|}
\hline wrt CC & CEO & Mo & MI \\
\hline CEO & EE & $\mathrm{MH}$ & $\mathrm{VH}$ \\
\hline $\mathrm{Mo}$ & & $\mathrm{EE}$ & $\mathrm{MH}$ \\
\hline $\mathrm{MI}$ & & & $\mathrm{EE}$ \\
\hline
\end{tabular}

\begin{tabular}{|c|c|c|c|}
\hline wrt CC & CEO & Mo & MI \\
\hline CEO & EE & H & AH \\
\hline Mo & & EE & MH \\
\hline MI & & & EE \\
\hline
\end{tabular}


End of Table 6

\begin{tabular}{|c|c|c|c|c|}
\hline \multicolumn{5}{|c|}{ Expert 1 } \\
\hline wrt OI & FJ & VO & T & Me \\
\hline FJ & EE & AH & H & AE \\
\hline VO & & EE & ML & AL \\
\hline T & & & EE & ML \\
\hline $\mathrm{Me}$ & & & & EE \\
\hline
\end{tabular}

\begin{tabular}{|c|c|c|c|c|}
\hline \multicolumn{5}{|c|}{ Expert 2 } \\
\hline wrt OI & FJ & VO & T & Me \\
\hline FJ & EE & VH & ML & L \\
\hline VO & & EE & ML & L \\
\hline T & & & EE & ML \\
\hline Me & & & & EE \\
\hline
\end{tabular}

\begin{tabular}{|c|c|c|c|c|}
\hline \multicolumn{5}{|c|}{ Expert 3 } \\
\hline wrt OI & FJ & VO & T & Me \\
\hline FJ & EE & MH & H & AE \\
\hline VO & & EE & MH & ML \\
\hline T & & & EE & L \\
\hline Me & & & & EE \\
\hline
\end{tabular}

\begin{tabular}{|c|c|c|c|c|}
\hline wrt F & A & 1 & FC & MU \\
\hline A & EE & H & VL & VL \\
\hline I & & EE & VL & L \\
\hline FC & & & EE & AE \\
\hline MU & & & & EE \\
\hline
\end{tabular}

\begin{tabular}{|c|c|c|c|c|}
\hline wrt F & A & I & FC & MU \\
\hline A & EE & VH & AH & MH \\
\hline I & & EE & MH & L \\
\hline FC & & & EE & VL \\
\hline MU & & & & EE \\
\hline
\end{tabular}

\begin{tabular}{|c|c|c|c|c|}
\hline wrt F & A & I & FC & MU \\
\hline A & EE & VH & VH & MH \\
\hline I & & EE & ML & VL \\
\hline FC & & & EE & L \\
\hline MU & & & & EE \\
\hline
\end{tabular}

Table 7. Aggregated evaluations for the model

\begin{tabular}{|l|l|l|l|l|l|l|l|l|l|l|l|l|l|l|l|l|}
\hline wrt goal & \multicolumn{4}{|c|}{ SC } & \multicolumn{4}{c|}{ CC } & \multicolumn{4}{c|}{ OI } & \multicolumn{3}{c|}{ F } \\
\hline SC & 0.500 & 0.500 & 0.500 & 0.500 & 0.552 & 0.703 & 0.171 & 0.297 & 0.415 & 0.559 & 0.325 & 0.441 & 0.460 & 0.652 & 0.232 & 0.348 \\
\hline CC & 0.186 & 0.301 & 0.548 & 0.699 & 0.500 & 0.500 & 0.500 & 0.500 & 0.217 & 0.317 & 0.533 & 0.683 & 0.304 & 0.445 & 0.422 & 0.555 \\
\hline OI & 0.367 & 0.498 & 0.368 & 0.502 & 0.534 & 0.684 & 0.215 & 0.316 & 0.500 & 0.500 & 0.500 & 0.500 & 0.600 & 0.615 & 0.232 & 0.385 \\
\hline F & 0.234 & 0.351 & 0.435 & 0.649 & 0.469 & 0.615 & 0.232 & 0.385 & 0.304 & 0.445 & 0.422 & 0.555 & 0.500 & 0.500 & 0.500 & 0.500 \\
\hline
\end{tabular}

\begin{tabular}{|c|c|c|c|c|c|c|c|c|c|c|c|c|}
\hline wrt SC & \multicolumn{4}{|c|}{ AOG } & \multicolumn{4}{c|}{ OS } & \multicolumn{4}{c|}{ RE } \\
\hline AOG & 0.500 & 0.500 & 0.500 & 0.500 & 0.600 & 0.750 & 0.100 & 0.250 & 0.460 & 0.652 & 0.232 & 0.348 \\
\hline OS & 0.100 & 0.250 & 0.600 & 0.750 & 0.500 & 0.500 & 0.500 & 0.500 & 0.217 & 0.317 & 0.533 & 0.683 \\
\hline RE & 0.234 & 0.351 & 0.435 & 0.649 & 0.534 & 0.684 & 0.215 & 0.316 & 0.500 & 0.500 & 0.500 & 0.500 \\
\hline
\end{tabular}

\begin{tabular}{|c|c|c|c|c|c|c|c|c|c|c|c|c|}
\hline wrt CC & \multicolumn{5}{|c|}{ CEO } & \multicolumn{4}{c|}{ Mo } & \multicolumn{5}{c|}{ MI } \\
\hline CEO & 0.500 & 0.500 & 0.500 & 0.500 & 0.534 & 0.684 & 0.215 & 0.316 & 0.499 & 0.665 & 0.000 & 0.335 \\
\hline Mo & 0.217 & 0.317 & 0.533 & 0.683 & 0.500 & 0.500 & 0.500 & 0.500 & 0.370 & 0.539 & 0.344 & 0.461 \\
\hline MI & 0.289 & 0.469 & 0.339 & 0.531 & 0.418 & 0.561 & 0.000 & 0.439 & 0.500 & 0.500 & 0.500 & 0.500 \\
\hline
\end{tabular}

\begin{tabular}{|c|c|c|c|c|c|c|c|c|c|c|c|c|c|c|c|c|}
\hline wrt OI & \multicolumn{4}{|c|}{$\mathrm{FJ}$} & \multicolumn{4}{|c|}{$\mathrm{VO}$} & \multicolumn{4}{|c|}{$\mathrm{T}$} & \multicolumn{4}{|c|}{$\mathrm{Me}$} \\
\hline FJ & 0.500 & 0.500 & 0.500 & 0.500 & 0.588 & 0.740 & 0.000 & 0.260 & 0.367 & 0.498 & 0.368 & 0.502 & 0.235 & 0.419 & 0.423 & 0.581 \\
\hline $\mathrm{VO}$ & 0.123 & 0.269 & 0.580 & 0.731 & 0.500 & 0.500 & 0.500 & 0.500 & 0.331 & 0.458 & 0.410 & 0.542 & 0.123 & 0.269 & 0.580 & 0.731 \\
\hline $\mathrm{T}$ & 0.415 & 0.559 & 0.325 & 0.441 & 0.447 & 0.591 & 0.292 & 0.409 & 0.500 & 0.500 & 0.500 & 0.500 & 0.250 & 0.334 & 0.516 & 0.666 \\
\hline $\mathrm{Me}$ & 0.466 & 0.622 & 0.229 & 0.378 & 0.588 & 0.740 & 0.000 & 0.260 & 0.517 & 0.668 & 0.232 & 0.332 & 0.500 & 0.500 & 0.500 & 0.500 \\
\hline
\end{tabular}

\begin{tabular}{|c|c|c|c|c|c|c|c|c|c|c|c|c|c|c|c|c|}
\hline wrt F & \multicolumn{4}{|c|}{ A } & \multicolumn{4}{|c|}{1} & \multicolumn{4}{|c|}{$\mathrm{FC}$} & \multicolumn{4}{|c|}{ MU } \\
\hline A & 0.500 & 0.500 & 0.500 & 0.500 & 0.584 & 0.734 & 0.126 & 0.266 & 0.499 & 0.665 & 0.000 & 0.335 & 0.392 & 0.549 & 0.335 & 0.451 \\
\hline I & 0.135 & 0.267 & 0.583 & 0.733 & 0.500 & 0.500 & 0.500 & 0.500 & 0.304 & 0.445 & 0.422 & 0.555 & 0.168 & 0.284 & 0.566 & 0.716 \\
\hline $\mathrm{FC}$ & 0.289 & 0.469 & 0.339 & 0.531 & 0.469 & 0.615 & 0.232 & 0.385 & 0.500 & 0.500 & 0.500 & 0.500 & 0.300 & 0.406 & 0.435 & 0.594 \\
\hline MU & 0.392 & 0.527 & 0.292 & 0.473 & 0.567 & 0.718 & 0.159 & 0.282 & 0.487 & 0.644 & 0.182 & 0.356 & 0.500 & 0.500 & 0.500 & 0.50 \\
\hline
\end{tabular}


To be able to illustrate the process briefly, sample tables and a calculation related to main criteria will be given in the following:

$$
\begin{aligned}
& \tilde{r}_{g_{12}}=\left(\left[\mu_{g_{12}}^{-}, \mu_{g_{12}}^{+}\right],\left[v_{g_{12}}^{-}, v_{g_{12}}^{+}\right]\right) ; \\
& \mu_{g_{12}}^{-}=1-(1-0.50)^{\frac{1}{3}} \times(1-0.55)^{\frac{1}{3}} \times(1-0.60)^{\frac{1}{3}}=0.552 ; \\
& \mu_{g_{12}}^{+}=1-(1-0.65)^{\frac{1}{3}} \times(1-0.70)^{\frac{1}{3}} \times(1-0.75)^{\frac{1}{3}}=0.703 ; \\
& v_{g_{12}}^{-}=(0.25 \times 0.20 \times 0.10)^{1 / 3}=0.171 ; \\
& v_{g_{12}}^{+}=(0.35 \times 0.30 \times 0.25)^{1 / 3}=0.297 . \\
& \text { Hence, } \tilde{r}_{g_{12}}=([0.552,0.703],[0.171,0.297]) .
\end{aligned}
$$

Step 3: The score judgment matrix $\tilde{S}=\left(\tilde{s}_{i j}\right)_{n \times n}$ and the interval multiplicative matrix $\tilde{A}=\left(\tilde{a}_{i j}\right)_{n \times n}$ are calculated using Eqs. (9) and (10), respectively. For the main criteria, score judgment matrices are given in Table $8 \mathrm{a}$, and interval multiplicative matrices are given in Table 8 b.

To illustrate the calculations, an example for the main criteria is given below.

$$
\begin{aligned}
\tilde{s}_{g_{12}} & =\left[\mu_{g_{12}}^{-}-v_{g_{12}}^{+}, \mu_{g_{12}}^{+}-v_{g_{12}}^{-}\right] ; \\
\tilde{s}_{g_{12}} & =[0.552-0.297,0.703-0.171]=[0.255,0.532], \text { and } \\
\tilde{a}_{g_{12}} & =\left[10^{0.255}, 10^{0.532}\right]=[1.797,3.403] .
\end{aligned}
$$

Step 4: The priority vectors of the interval multiplicative matrices related to main and subcriteria are determined by using Eq. (11) where TOTAL- is $\sum_{j=1}^{n} \tilde{a}_{i j}^{-}$, and TOTAL+ is $\sum_{j=1}^{n} \tilde{a}_{i j}^{+}$. The
results for the main criteria are shown in Table 8c.

To calculate the values for SC in the main criteria matrix, for example;

TOTAL- $=1.000+1.797+0.942+1.295=5.035$ (Sum of column “-” values of raw "SC" in Table 8c).

TOTAL+ $=1.000+3.403+1.713+2.632=8.748$ (Sum of column “+” values of raw "SC" in Table 8c).

Weight $-=5.035 / 24.983=0.202$ (TOTAL - of SC divided by sum of all TOTAL + in the matrix).

Weight $+=8.768 / 15.432=0.567$ (TOTAL + of SC divided by sum of all TOTAL - in the matrix).

Step 5: The possibility degree matrices are constructed using Eq. (12). Results for the main criteria can be found in Table $8 \mathrm{~d}$.

In the calculation of $1^{\text {st }}$ raw $2^{\text {nd }}$ column value of the main criteria matrix, for example,

$P\left(w_{S C} \geq w_{C C}\right)=\frac{\min \left\{L_{w_{S C}}+L_{w_{C C}}, \max \left(w_{S C}{ }^{+}-w_{C C}-0\right)\right\}}{L_{w_{S C}}+L_{w_{C C}}}$,

$L_{w_{S C}}=w_{S C}^{+}-w_{S C}^{-}=0.5669-0.2015=0.3654$ and 


$$
\begin{aligned}
& L_{w_{C C}}=w_{C C}^{+}-w_{C C}^{-}=0.2093-0.0885=0.1208 . \text { So; } \\
& P\left(w_{S C} \geq w_{C C}\right)=\frac{\min \{0.3654+0.1208, \max (0.5669-0.0885,0)\}}{0.3654+0.1208}=0.984 .
\end{aligned}
$$

Table 8. Matrices for main criteria in intermediate calculation steps with aggregated evaluations a) The score judgement matrix

\begin{tabular}{|c|c|c|c|c|c|c|c|c|}
\hline \multirow{2}{*}{ wrt goal } & \multicolumn{2}{|c|}{ SC } & \multicolumn{2}{c|}{ CC } & \multicolumn{2}{c|}{ OI } & \multicolumn{2}{c|}{ F } \\
\hline SC & 0.000 & 0.000 & 0.255 & 0.532 & -0.026 & 0.234 & 0.112 & 0.420 \\
\hline CC & -0.513 & -0.247 & 0.000 & 0.000 & -0.466 & -0.216 & -0.251 & 0.024 \\
\hline OI & -0.135 & 0.129 & 0.218 & 0.469 & 0.000 & 0.000 & 0.215 & 0.383 \\
\hline F & -0.415 & -0.084 & 0.084 & 0.383 & -0.251 & 0.024 & 0.000 & 0.000 \\
\hline
\end{tabular}

b) The interval multiplicative matrix

\begin{tabular}{|c|c|c|c|c|c|c|c|c|}
\hline \multirow{2}{*}{ wrt goal } & \multicolumn{2}{|c|}{ SC } & \multicolumn{2}{c|}{ CC } & \multicolumn{2}{c|}{ OI } & \multicolumn{2}{c|}{ F } \\
\cline { 2 - 9 } & - & + & - & + & - & + & - & + \\
\hline SC & 1.000 & 1.000 & 1.797 & 3.403 & 0.942 & 1.713 & 1.295 & 2.632 \\
\hline CC & 0.307 & 0.566 & 1.000 & 1.000 & 0.342 & 0.609 & 0.561 & 1.056 \\
\hline OI & 0.733 & 1.347 & 1.652 & 2.943 & 1.000 & 1.000 & 1.642 & 2.417 \\
\hline F & 0.385 & 0.824 & 1.214 & 2.417 & 0.561 & 1.056 & 1.000 & 1.000 \\
\hline
\end{tabular}

c) The priority vectors of the interval multiplicative matrix

\begin{tabular}{|c|c|c|c|c|}
\cline { 3 - 5 } \multicolumn{1}{c|}{} & \multirow{2}{*}{ Total -} & \multirow{2}{*}{ Total +} & \multicolumn{2}{c|}{ Weight } \\
\cline { 4 - 5 } \multicolumn{1}{c|}{} & & & - & + \\
\hline SC & 5.035 & 8.748 & 0.202 & 0.567 \\
\hline CC & 2.210 & 3.230 & 0.088 & 0.209 \\
\hline OI & 5.028 & 7.707 & 0.201 & 0.499 \\
\hline F & 3.159 & 5.297 & 0.126 & 0.343 \\
\hline$\sum=$ & 15.432 & 24.983 & \multicolumn{2}{|c}{} \\
\cline { 1 - 4 }$\sum=$
\end{tabular}

d) The possibility degree matrix

\begin{tabular}{|c|c|c|c|c|}
\cline { 2 - 5 } \multicolumn{1}{c|}{} & SC & CC & OI & F \\
\hline SC & 0.500 & 0.984 & 0.551 & 0.757 \\
\hline CC & 0.016 & 0.500 & 0.019 & 0.245 \\
\hline OI & 0.449 & 0.981 & 0.500 & 0.724 \\
\hline F & 0.243 & 0.755 & 0.276 & 0.500 \\
\hline
\end{tabular}


Step 6: The possibility degree matrices are prioritized using Eq. (13). The resultant priority values for the main criteria are $0.948,0.445,0,913$, and 0.693 for SC, CC, OI, and F, respectively. The priority value of SC in the main criteria, for example, is calculated as shown below:

$$
w_{S C}=\frac{1}{4}\left[\sum_{j=1}^{4} p_{S C j}+\frac{4}{2}-1\right]=\frac{1}{4}[0.500+0.984+0.551+0.757+2-1]=0.948 \text {. }
$$

Step 7: The weights vectors obtained in the previous stage are normalized giving the local weights of the criteria and sub-criteria. The local weight of each sub-criterion is then multiplied by the weight of the corresponding main-criterion to end up with the global weights. The results are presented in Table 9.

Table 9. Final weights of main and sub-criteria for aggregated evaluations

\begin{tabular}{|l|c|c|c|c|c|c|c|c|c|c|c|c|c|c|}
\cline { 2 - 15 } \multicolumn{1}{c|}{} & \multicolumn{3}{c|}{ SC } & \multicolumn{3}{c|}{ CC } & \multicolumn{4}{c|}{ OI } & \multicolumn{4}{c|}{ F } \\
\cline { 2 - 15 } & \multicolumn{3}{c|}{0.316} & \multicolumn{3}{c|}{0.148} & \multicolumn{3}{c|}{0.304} & \multicolumn{4}{c|}{0.231} \\
\cline { 2 - 15 } & AOG & OS & RE & CEO & Mo & MI & FJ & VO & T & Me & A & I & FC & MU \\
\hline $\begin{array}{l}\text { Local } \\
\text { weights }\end{array}$ & 0.478 & 0.156 & 0.365 & 0.420 & 0.235 & 0.345 & 0.290 & 0.151 & 0.230 & 0.329 & 0.320 & 0.147 & 0.232 & 0.301 \\
\hline $\begin{array}{l}\text { Global } \\
\text { weights }\end{array}$ & 0.151 & 0.049 & 0.115 & 0.062 & 0.035 & 0.051 & 0.088 & 0.046 & 0.070 & 0.100 & 0.074 & 0.034 & 0.054 & 0.070 \\
\hline
\end{tabular}

The final weight of SC, for example, can be calculated by using normalization as:

$$
w_{S C}=\frac{0.948}{3}=0.316 \text {. }
$$

\subsection{Analysis based on compromised assessments}

Step 1. The compromised linguistic pairwise comparison matrices of the criteria given in Figure 1 are obtained as in Table 10.

Table 10. Compromised linguistic pairwise comparison matrices for the main and sub-criteria

\begin{tabular}{|l|c|c|c|c|}
\hline wrt goal & SC & CC & OI & F \\
\hline SC & EE & H & MH & H \\
\hline CC & & EE & L & AE \\
\hline OI & & & EE & H \\
\hline F & & & & EE \\
\hline
\end{tabular}

\begin{tabular}{|c|c|c|c|c|c|c|c|c|}
\hline wrt SC & AOG & OS & RE & & wrt CC & CEO & Mo & MI \\
\hline AOG & EE & VH & H & & CEO & EE & H & MH \\
\hline OS & & EE & ML & & Mo & & EE & ML \\
\hline RE & & & EE & & MI & & & EE \\
\hline
\end{tabular}


End of Table 10

\begin{tabular}{|c|c|c|c|c|c|c|c|c|c|c|}
\hline wrt OI & FJ & VO & T & Me & & wrt F & A & I & FC & MU \\
\hline FJ & $\mathrm{EE}$ & $\mathrm{VH}$ & $\mathrm{AE}$ & $\mathrm{L}$ & & $\mathrm{A}$ & $\mathrm{EE}$ & $\mathrm{H}$ & $\mathrm{MH}$ & $\mathrm{AE}$ \\
\hline $\mathrm{VO}$ & & $\mathrm{EE}$ & $\mathrm{L}$ & $\mathrm{VL}$ & & $\mathrm{I}$ & & $\mathrm{EE}$ & $\mathrm{ML}$ & $\mathrm{L}$ \\
\hline $\mathrm{T}$ & & & $\mathrm{EE}$ & $\mathrm{L}$ & & $\mathrm{FC}$ & & & $\mathrm{EE}$ & $\mathrm{ML}$ \\
\hline $\mathrm{Me}$ & & & & $\mathrm{EE}$ & & $\mathrm{MU}$ & & & & $\mathrm{EE}$ \\
\hline
\end{tabular}

Step 2. The linguistic data are then converted to their corresponding interval-valued intuitionistic fuzzy sets using Table 1.

Step 3 thru 7 are computationally the same with the previous case. So, to keep the study concise, these calculations are not given here. The final results are presented in Table 11.

Table 11. Final weights of main and sub-criteria for compromised evaluations

\begin{tabular}{|l|c|c|c|c|c|c|c|c|c|c|c|c|c|c|}
\cline { 2 - 16 } \multicolumn{1}{c|}{} & \multicolumn{3}{c|}{ SC } & \multicolumn{3}{c|}{ CC } & \multicolumn{4}{c|}{ OI } & \multicolumn{4}{c|}{ F } \\
\cline { 2 - 15 } & \multicolumn{3}{c|}{0.337} & \multicolumn{3}{c|}{0.198} & \multicolumn{3}{c|}{0.305} & \multicolumn{4}{c|}{0.159} \\
\cline { 2 - 15 } & AOG & OS & RE & CEO & Mo & MI & FJ & VO & T & Me & A & I & FC & MU \\
\hline $\begin{array}{l}\text { Local } \\
\text { weights }\end{array}$ & 0.506 & 0.162 & 0.332 & 0.478 & 0.169 & 0.352 & 0.289 & 0.118 & 0.246 & 0.347 & 0.322 & 0.140 & 0.232 & 0.307 \\
\hline $\begin{array}{l}\text { Global } \\
\text { weights }\end{array}$ & 0.171 & 0.055 & 0.112 & 0.095 & 0.034 & 0.070 & 0.088 & 0.036 & 0.075 & 0.106 & 0.051 & 0.022 & 0.037 & 0.049 \\
\hline
\end{tabular}

\section{Results and discussion}

Table 9 and Table 11 indicate that the aggregated and compromised assessments yield different results. In the aggregated results, the main criteria are ranked as $\mathrm{SC}>\mathrm{OI}>\mathrm{F}>\mathrm{CC}$, whereas in the compromised results it is $\mathrm{SC}>\mathrm{OI}>\mathrm{CC}>\mathrm{F}$. In the rankings of the subcriteria, although the first three items remain unchanged, there are significant changes in the positions of some others. In the aggregated case, the ranking is $\mathrm{AOG}>\mathrm{RE}>\mathrm{Me}>\mathrm{FJ}>\mathrm{A}$ $>\mathrm{T}=\mathrm{MU}>\mathrm{CEO}>\mathrm{FC}>\mathrm{MI}>\mathrm{OS}>\mathrm{VO}>\mathrm{Mo}>\mathrm{I}$ whereas it is $\mathrm{AOG}>\mathrm{RE}>\mathrm{Me}>\mathrm{CEO}>$ $\mathrm{FJ}>\mathrm{T}>\mathrm{MI}>\mathrm{OS}>\mathrm{A}>\mathrm{MU}>\mathrm{FC}>\mathrm{VO}>\mathrm{Mo}>\mathrm{I}$ in the compromised case. These findings justify our hypothesis, which the results of aggregated and compromised assessments may change. Since, there was not the groupthink effect in our case, and all of the experts were happy with the compromised assessments at the end of the discussions, the preferred solution is taken as the compromised solution as shown in Table 11.

Performance Management is one of the most important functions of the HRM practices. Alignment of HR practices with the strategic objectives is pivotal in achieving its strategic goals. The role and perspective executives have over the performance management system facilitate its effectiveness. In line with these, the compromised results of the main criteria, with a slight variation to the aggregated one, show that Strategic Congruence (SC) ranks as highest. A strategically congruent performance management system is an enabler for leading companies' short or long-term strategy. Every involved individual, whether appraiser or 
appraisee, ought to be thoroughly part of the management process; share the willingness for a just and fair management system; and use a valid and measurable tool for objectivity assurance. Vitality of a performance management system in comparison to other HRM functions is that, HR department may take the leadership of managing all other functions, whereas, managers involved have the obligation and willingness to take effective and active role in its objective implementation. Parallel to above, results indicate Objective Implementation (OI) as second most important criterion for an effective performance management system. Results further show that, Contextual Congruence (CC) ranks as third priority. Once strategical congruence and objective implementation is assured, individuals need to be culturally engaged to using and housing a performance management system. An effective performance management system may be established provided that all members have an organizational identity and motivation towards a performance management process, and assuming a positive attitude in choosing the implementation methods.

When the compromised rankings of the sub-criteria are analysed, results are quite distinctive from the aggregated ones. Alignment with organizational goals (AOG) and role of the executive (RE) - the sub-dimensions of SC, rank at first and second degree. For effectiveness of a performance management system, the role and leadership of the executives is pivotal; both in identifying and in linking the individual goals with organization's strategic goals. Further, a bias-free measurement, through systematic process delivering objective and meaningful results are ranked as third prioritized sub-criteria. An effective performance management system needs to deliver measurable and manageable results. Peter Drucker's famous quote, saying "if you cannot measure it, you cannot manage it" are once again justified as among main priorities. Additionally, these three first ranking sub-criteria have priority weights above 0.1 each within the model.

\section{Conclusions}

Establishing an effective performance management process is a challenging issue for organizations. Specially, when complex and vague factors are involved, managers need to take all of them into account and pick the most functional and applicable model. However, a model that is derived based on only a sub-set of important criteria, through limited cognitive ability, will ignore the relative connection and interaction among them. Thus, a fuzzy analytic hierarchy (AHP) based evaluation process may facilitate managers, by synthesizing their data and delivering a more rational decision making.

The proposed model can take different experts' intuitionistic assessments into account in both aggregated and compromised assessments. Thus the model has the ability to consider both vagueness and diversity in human reasoning. It is applicable even if you do not have any tangible attributes. It can be used when all the evaluation data are linguistic. An MS Excel work- sheet easily allows you to formulate the whole process.

This paper showed how the criteria for an effective performance management system are weighed in the presence of intuitionistic fuzzy assessments. The weights of the criteria are obtained by both aggregation of individual assessments and compromised assessments of experts to question any differences in between. The results exhibit a difference between the 
final ranking results of the two methods. Thus, in a multi-expert decision, aggregation and compromised solutions cannot be used interchangeably and the most appropriate method needs to be chosen considering the case at hand.

Our suggestions for further research are to use different aggregation operations for interval-valued intuitionistic fuzzy sets or to use different extension types of fuzzy sets to represent the vagueness in the assessments. For instance, triangular intuitionistic fuzzy sets for the representation and different aggregation operators for this type of fuzzy sets can be used.

\section{References}

Aguinis, H. (2009). Performance management (2nd ed.). Upper Saddle River, NJ: Pearson Prentice Hall.

Aguinis, H., Joo, H., \& Gottfredson, R. K. (2011). Why we hate performance management - And why we should love it? Journal of Business Horizons, 54(6), 503-507.

https://doi.org/10.1016/j.bushor.2011.06.001

Altarawneh, I. I. (2016). Strategic human resources management and its impact on performance: the case from Saudi Arabia. International Journal of Business Management and Economic Research, 7(1), 486-503.

Armstrong, M. (2000). Performance management: Key strategies and practical guidelines. London, UK: Kogan Page Limited.

Arnaboldi, M., Lapsley, I., \& Steccolini, I. (2015). Performance management in the public sector: The ultimate challenge. Journal of Financial Accountability and Management, 32(1), 1-22. https://doi.org/10.1111/faam.12049

Atanassov, K. (1983). Intuitionistic fuzzy sets, VII ITKR's session. Sofia (Deposed in Central ScienceTechnical Library of Bulgarian Academy of Sciences 1697/84) (in Bulgarian).

Atanassov, K. T. (1986). Intuitionistic fuzzy sets. Fuzzy Sets and Systems, 20(1), 87-96. https://doi.org/10.1016/S0165-0114(86)80034-3

Bach, S. (2005). New directions in performance management (Chapter 11). In S. Bach (Ed.), Managing human resources: personnel management in transition. Oxford: Blackwell.

Balin, A., \& Baraçli, H. (2015). A fuzzy multi-criteria decision making methodology based upon the interval type-2 fuzzy sets for evaluating renewable energy alternatives in Turkey. Technological and Economic Development of Economy, 1-22. https://doi.org/10.3846/20294913.2015.1056276

Boswell, W. R. (2006). Aligning employees with the organization's strategic objectives: Out of "line of sight", out of mind. International Journal of Human Resource Management, 17(9), 1489-1511. https://doi.org/10.1080/09585190600878071

Boswell, W. R., Bingham, J. B., \& Colvin, A. J. S. (2006). Aligning employees through "line of sight". Business Horizons, 49(6), 499-509. https://doi.org/10.1016/j.bushor.2006.05.001

Bouskila-Yam, O., \& Kluger, A. N. (2011). Strength-based performance appraisal and goal setting. Human Resource Management Review, 21(2), 137-147. https://doi.org/10.1016/j.hrmr.2010.09.001

Buller, P. F., \& McEnvoy, G. M. (2012). Strategy, human resources management and performance: Sharpening line of sight. Human Resource Management Review, 22(1), 43-56. https://doi.org/10.1016/j.hrmr.2011.11.002

Cardy, R. L., \& Leonard, B. (2011). Performance management: concepts, skills and exercises (2nd ed.). Armonk, NY: M. E. Sharpe.

Chadwick, C. (2010). Theoretic insights on the nature of performance synergies in human resource systems: Toward greater precision. Human Resource Management Review, 20(2), 85-101.

https://doi.org/10.1016/j.hrmr.2009.06.001 
Chen, T. Y. (2014). A prioritized aggregation operator-based approach to multiple criteria decision making using interval-valued intuitionistic fuzzy sets: A comparative perspective. Information Sciences, 281, 97-112. https://doi.org/10.1016/j.ins.2014.05.018

Chen, T. Y. (2017). Multiple criteria decision analysis using prioritised interval type-2 fuzzy aggregation operators and its application to site selection. Technological and Economic Development of Economy, 23(1), 1-21. https://doi.org/10.3846/20294913.2016.1209249

Chytas, P., Glykas, M., \& Valiris, G. (2011). A proactive balanced scorecard. International Journal of Information Management, 31, 460-468. https://doi.org/10.1016/j.ijinfomgt.2010.12.007

Datta, D. K., Guthrie, J. P., \& Wright, P. M. (2005). Human resource management and labor productivity: Does industry matter? Academy of Management Journal, 48(1), 135-145. https://doi.org/10.5465/amj.2005.15993158

Erdogan, B. (2002). Antecedents and consequences of justice perceptions in performance appraisals. Human Resources Review, 12(4), 555-578. https://doi.org/10.1016/S1053-4822(02)00070-0

Gruman, J. A., \& Saks, A. M. (2011). Performance management and employee engagement. Human Resource Management Review, 21(2), 123-136. https://doi.org/10.1016/j.hrmr.2010.09.004

Guest, D. E. (2011). Human resource management and performance: Still searching for some answers. Human Resource Management Journal, 21(1), 3-13. https://doi.org/10.1111/j.1748-8583.2010.00164.x

Hajiagha, S. H. R., Hashemi, S. S., \& Zavadskas, E. K. (2013). A complex proportional assessment method for group decision making in an interval-valued intuitionistic fuzzy environment. Technological and Economic Development of Economy, 19(1), 22-37. https://doi.org/10.3846/20294913.2012.762953

Jiang, K., Lepak, D. P., Han, K., Hong, Y., Kim, A., \& Winkler, A. L. (2012). Clarifying the construct of human resource systems: Relating human resource management to employee performance. Human Resource Management Review, 22(2), 73-85. https://doi.org/10.1016/j.hrmr.2011.11.005

Lepak, D. P., Liao, H., Chung, Y., \& Harden, E. E. (2006). A conceptual review of human resource management systems in strategic human resource management research (Chapter 7). In J. J. Matocchio (Ed.), Research in personnel and human resource management (Vol. 25, p. 217-271). Greenwich, CT: JAI Press. https://doi.org/10.1016/S0742-7301(06)25006-0

Liu, P., Li, Y., \& Antuchevičienė, J. (2016). Multi-criteria decision-making method based on intuitionistic trapezoidal fuzzy prioritised OWA operator. Technological and Economic Development of Economy, 22(3), 453-469. https://doi.org/10.3846/20294913.2016.1171262

Mone, E. M., \& London, M. (2010). Employee engagement through effective performance management: A practical guide for managers. New York, NY: Routledge.

Moona, S. H., Scullenb, S. E., \& Lathamc, G. P. (2016). Precarious curve ahead: The effects of forced distribution rating systems on job performance. Human Resource Management Review, 26(2), 166179. https://doi.org/10.1016/j.hrmr.2015.12.002

Paauwe, J., \& Boselie, P. (2005). HRM and performance: What next? Human Resource Management Journal, 15(4), 68-83. https://doi.org/10.1111/j.1748-8583.2005.tb00296.x

Prowse, P., \& Prowse, J. (2009). The dilemma of performance appraisal. Measuring Business Excellence, 13(4), 69-77. https://doi.org/10.1108/13683040911006800

Taylor, J. (2014). Organizational Culture and the paradox of performance management. Journal of Public Performance and Management Review, 38(1), 7-22. https://doi.org/10.2753/PMR1530-9576380101

Wei, G., Zhao, X., \& Wang, H. (2012). An approach to multiple attribute group decision making with interval intuitionistic trapezoidal fuzzy information. Technological and Economic Development of Economy, 18(2), 317-330. https://doi.org/10.3846/20294913.2012.676995

$\mathrm{Xu}, \mathrm{Z}$. S., \& Da, Q. L. (2003). Possibility degree method for ranking interval numbers and its application. Journal of System Engineering, 18(1), 67-70. 
Yang, W., Chen, Z., \& Zhang, F. (2017). New group decision making method in intuitionistic fuzzy setting based on TOPSIS. Technological and Economic Development of Economy, 23(3), 441-461. https://doi.org/10.3846/20294913.2015.1072754

Youndt, M. A., Snell, S. A., Dean, J. W., \& Lepak, D. P. (1996). Human resource management, manufacturing strategy, and firm performance. Academy of Management Journal, 39(4), 836-866.

Yu, D. (2013). Intuitionistic fuzzy prioritized operators and their application in multi-criteria group decision making. Technological and Economic Development of Economy, 19(1), 1-21. https://doi.org/10.3846/20294913.2012.762951

Zavadskas, E. K., Bausys, R., Kaklauskas, A., Ubarte, I., Kuzminskė, A., \& Gudienė, N. (2017). Sustainable market valuation of buildings by the single-valued neutrosophic MAMVA method. Applied Soft Computing, 57, 74-87. https://doi.org/10.1016/j.asoc.2017.03.040

Zavadskas, E. K., Bausys, R., \& Lazauskas, M. (2015). Sustainable assessment of alternative sites for the construction of a waste incineration plant by applying WASPAS method with single-valued neutrosophic set. Sustainability, 7(12), 15923-15936. https://doi.org/10.3390/su71215792

Zhao, H., Xu, Z., Ni, M., \& Liu, S. (2010). Generalized aggregation operators for intuitionistic fuzzy sets. International Journal of Intelligent Systems, 25(1), 1-30. https://doi.org/10.1002/int.20386

Zhou, L., Tao, Z., Chen, H., \& Liu, J. (2014). Continuous interval-valued intuitionistic fuzzy aggregation operators and their applications to group decision making. Applied Mathematical Modelling, 38(7-8), 2190-2205. https://doi.org/10.1016/j.apm.2013.10.036 